\title{
Some Mechanical and Thermal Properties of PC/ABS Blends
}

\author{
Rachida Krache, Ismahane Debbah
}

LMPMP, Département de Génie des Procédés, Faculté de Technologie, Université Ferhat Abbas, Sétif, Algérie. Email: rachida2000fr@yahoo.fr

Received March $9^{\text {th }}, 2011$; revised March 16 $6^{\text {th }}, 2011$; accepted March $30^{\text {th }}, 2011$.

\begin{abstract}
A series of blends of Acrylonitrile-Butadiene-Styrene (ABS) and Polycarbonate (PC) were prepared and some of their thermal and mechanical properties were determined. The Young's modulus changed gradually and monotonically with the polycarbonate content. This effect was tentatively explained as the antiplasticization of the PC which is ascribed to the chain mobility, which permits the PC chains to pack more tightly, to the secondary cross-linking between the PC chains, or to the secondary attachment of bulky side-chains to the PC, thus producing steric hindrance to the rotation of the PC main chains. The experimental values found for the impact strength were intermediate between those of the neat polymers, depending upon the dispersed rubber particles of butadiene in the matrix of SAN (Styrene-Acrylonitrile), and the dispersed PC particles which generally make the ABS more brittle. A maximum value of about $88 \mathrm{KJ} / \mathrm{m}^{2}$ for the impact strength was observed for the blend with $90 \%$ PC. This may be attributed to the strong polymer-polymer interactions for this particular composition. The variations in the heat deflection temperature HDT and the Vicat softening point with the blend composition were very similar, and allowed us to assume that the phase inversion between the matrices of the two polymers takes place at 50\% PC. The morphology of the blends revealed by SEM observation, show a co-continuous structure.
\end{abstract}

Keywords: PC, ABS, Blends, Impact Strength, Tensile and Flexural Properties, HDT, Vicat Point, Hardness, FTIR, Morphology

\section{Introduction}

The mixing or blending of polymers can be an efficient way of developing materials with novel or selectively enhanced properties. It is possible to modify some characteristics of polymer blends by varying the composition. Incompatible blends present phase separation as dispersed nodules in the matrix phase, the consequent low adhesion leading to undesirable properties. By controlling the fraction of each component, a resulting polymer with the desired properties can be obtained. Usually, the final objective is to reach a balance between maximum temperature resistance, toughness, etc, and ease of fabrication. The behavior of polymer blends can be discussed according to the variation of their properties with the blend composition. The blending of two or more polymers always affects the properties of the resulting material. Three different effects on those properties can be distinguished: synergistic, antagonistic and additive effect.
Polycarbonate PC is characterized for its high modulus, high toughness, high impact strength and difficult processability, due to its high melt viscosity. AcrylonitrileButadiene-Styrene (ABS) is a rubber toughened thermoplastic, characterized by its notch insensitivity and low cost. On the other hand, the ABS limitations are: poor flame and chemical resistance, and low thermal stability [1].

The commercialization of PC began in 1958; the production of the PC/ABS blends started on 1977. The addition of ABS to PC minimizes its drawbacks without affecting its superior mechanical properties, and also generates other useful characteristics, such as glossiness and low temperature toughness [2]. A number of patents concerning these blends have been issued in the past, but the scientific knowledge about their behavior is still limited owing to the complexity of the system. The blends consist, in fact, of four polymeric species and three phases, their morphology depending on a variety of factors: molecular characteristics of the components, ABS 
composition, blend composition, rheological properties, processing conditions, thermal treatments, etc.

$\mathrm{PC}$ and ABS are fairly similar in polarity, and might be compatible with each other; the ABS grafted rubber (butadiene) particles chains would remain insoluble, but firmly bonded by their styrene-acrylonitrile side-chains, producing good physical properties [3]. Assuming this fundamental basis for semi-compatibility, it is interesting to determine simultaneously the behavior of the modulus and the impact strength of the blends versus the PC content. The objective of the current study is to investigate the optimal composition of PC/ABS blend showing the highest value in different properties without adding any comptabilizer to this blend.

In the present paper we report the results obtained in the mechanical and thermal characterization of the PC/ABS blends. Thus, the bending, tensile, hardness and impact strength tests, as well as the heat deflection and distortion temperature tests were performed in the $\mathrm{PC} /$ ABS blends, and the results obtained were correlated with the blends composition.

\section{Experimental}

\subsection{Materials and Blend Preparation}

The materials used in this investigation were the following:

Polycarbonate PC Makrolon 3100, supplied by Bayer.

Acrylonitrile-Butadiene-Styrene (ABS) terpolymer Terluran, supplied by BASF.

Both polymers were dried in vacuum at $100^{\circ} \mathrm{C}(\mathrm{PC})$ and $70^{\circ} \mathrm{C}(\mathrm{ABS})$ for a period of $24 \mathrm{~h}$ before processing.

Dry PC/ABS mixtures with 100/0, 90/10, 80/20, 70/30, $60 / 40,50 / 50,40 / 60,30 / 70,20 / 80,10 / 90$ and $0 / 100$ weight compositions were prepared in a two roller mixer Polymix $80 \mathrm{~T}$ (Schwabenthan) at $220^{\circ} \mathrm{C}$ for 10 minutes Thereafter, the pelletized blends were mould compressed for $3 \mathrm{~min}$ in a hydraulic press at $250^{\circ} \mathrm{C}$ and $200 \mathrm{~kg} / \mathrm{cm}^{2}$.

\subsection{Techniques}

Tensile test on PC/ABS blends (ISO-R-527 standard) were carried out at room temperature using an AdamelLhomargy DY25 testing machine, assisted with a computer. The force sensor was 2000 Newton and a crosshead speed of $20 \mathrm{~mm} / \mathrm{min}$ was used.

Flexural test was carried out in three-point bending on the same machine of the tensile test at a cross head speed of $5 \mathrm{~mm} / \mathrm{min}$. The specimen dimensions were $4 \mathrm{~mm}$ in thickness and $10 \mathrm{~mm}$ in width. The distance between the supports was $100 \mathrm{~mm}$.

Izod impact test was performed on notched specimens (method A, ASTM.D 256-73 standard) using a CEAST pendulum, equipped with a 7.5 Joule hammer.
The hardness shore D test (ASTM D2240 standard) was performed using a conventional testing machine apparatus and a load of $5 \mathrm{~kg}$.

Vicat softening temperatures were determined according to ASTMD 648-72 method A, using a Heraeus Hanalt apparatus.

The heat deflection temperature (HDT) was calculated according to ISO-R-75 method A, using a DAVENPORT equipment.

Fourier transform infrared (FTIR) spectra, of compression molded thin films were recorded on a Perkin Elmer Infrared spectroscopy.

The fractured surface of compression molded specimens (of Notched Impact testing) were coated, and etched (to have a phase contrast) with an aqueous sodium hydroxide solution ( $35 \mathrm{wt} \%$ soda) at $110^{\circ} \mathrm{C}$ for about 20 minutes to dissolve the PC phase. The specimens were examined in a LEO 435 VP Scanning Electron Microscopy (SEM).

\section{Results and Discussion}

\subsection{Tensile Test}

The variation of Young's modulus in PC/ABS blends as a function of composition is illustrated in Figure 1. It can be observed that the experimental values follow the additivity law, as expected from the general composite theories. Thus, the modulus of the PC/ABS blends appears to vary gradually and monotonically from the value of the ABS to that of the PC. As it is commonly found, the modulus progressively decreases as the ABS content is increased, due to the influence of the ABS rubber component. The decrease has been postulated to be the tighter molecular packing revealed by the lowering in the density data as mentioned in [4] and was tentatively explained as the anti plasticization of the $\mathrm{PC}$ which is ascribed to the chain mobility, which permits the PC chains to pack more tightly, to the secondary cross-linking between the PC chains, or to the secondary attachment of bulky side-chains to the PC, thus producing steric hindrance to the rotation of the PC main chains [5].

In Figure 2, it can be observed that the stress-at-break composition curves show negative deviation, (blend properties lie below the additives line up to $100 \%$ PC content), except for the blend containing $90 \%$ PC were a maximum is again obtained. The observed negative deviation is due to the poor interfacial adhesion between the homopolymers phases, which causes poor stress transfer between the matrix and the dispersed phase. A little decrease in the slope of the stress properties composition curve is seen between the composition range $(0-30 \%$ $\mathrm{PC}$ ), and then an increase is observed with PC which is due to the higher proportion of the hard plastic phase. 


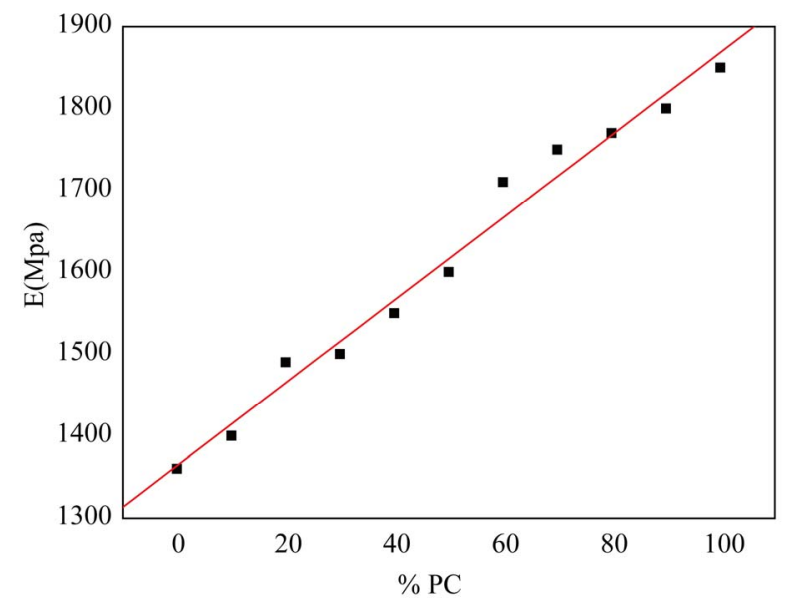

Figure 1. Young Modulus of PC/ABS blends as a function of the PC content.

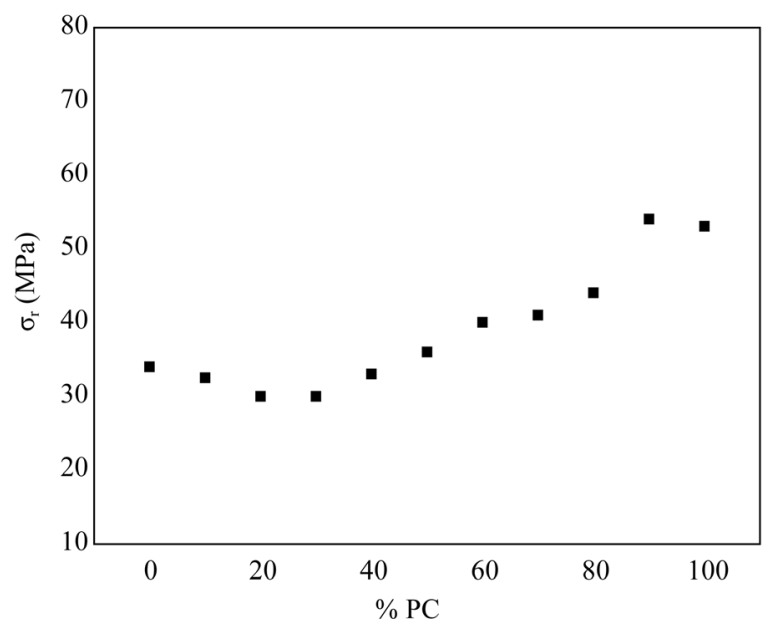

Figure 2. Stress at break of PC/ABS blends as a function of the PC content.

The effect of the mixing on the break strain of the blends is even more important than that observed in the break stress, and it is illustrated in Figure 3. A dramatic reduction of this property is observed in the mixtures with respect to linearity until the PC content reaches $30 \%$. Then, the break strain remains constant for the blends with $30 \%$ to $60 \%$ PC. Thereafter, a speedy rise in the values of the break strain is observed, with a maximum appearing again for the sample with $90 \%$ PC. The extreme influence of the incompatibility in the deformation at break of polymer blends is well known. Incompatibility gives rise to deformations at break on the order of $1 \%$. Thus, the ductility level of the PC/ABS blends can be a consequence of the combined effect of the rubbery behavior of the butadiene chains in the ABS and the partial compatibility between the two polymers at $90 \%$ PC.

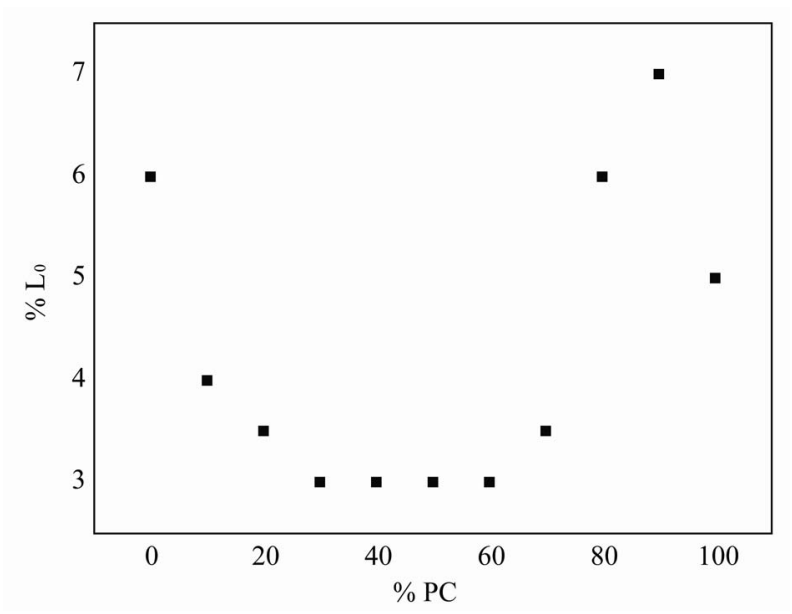

Figure 3. Strain at break of PC/ABS blends as a function of the PC content.

\subsection{Flexural Properties}

Both the flexural modulus and the strength of the blends have been measured. In Figure 4, the values of the flexural elastic modulus measured in three-point bending exhibit a clear positive deviation from linearity, that is, a synergistic behavior. Thus, all the $\mathrm{E}$ values obtained for the blends with $60 \%, 70 \%, 80 \%$ and $90 \%$ PC are higher than those obtained for the pure components of the blends. It can be supposed that the component of highest modulus contributes to the resulting value in a greater extent than the corresponding to the composition. The stress or strain concentrations in the matrix may give rise to increased local contributions to the overall stress with respect to that which corresponds to the composition of the blend [6]. Synergistic behavior in the modulus of polymer blends in relation with composition has been explained in some cases as a consequence of the blend densification, due to interactions between the components [6]. In Figure 5, the flexural break stress appears to vary gradually and monotonically with the PC content, while a significant decrease in the strain is observed in Figure 6. However, a maximum strain value is again obtained for the blend with $90 \%$ PC.

\subsection{Impact Strength}

The level of the notched impact strength for both neat polymers was rather poor (see Figure 7). Due to their incompatibility, the blends exhibited impact strength even lower to the ones showed by pure components. The result of the Izod impact testing shows the same features as shown by elongation-at-break (Figure 3). While blends with lower ABS content (10\%) exhibit high impact strength value $\left(88 \mathrm{~kJ} / \mathrm{m}^{2}\right)$, ABS rich blends have much smaller impact strength values than, both, pure PC and 


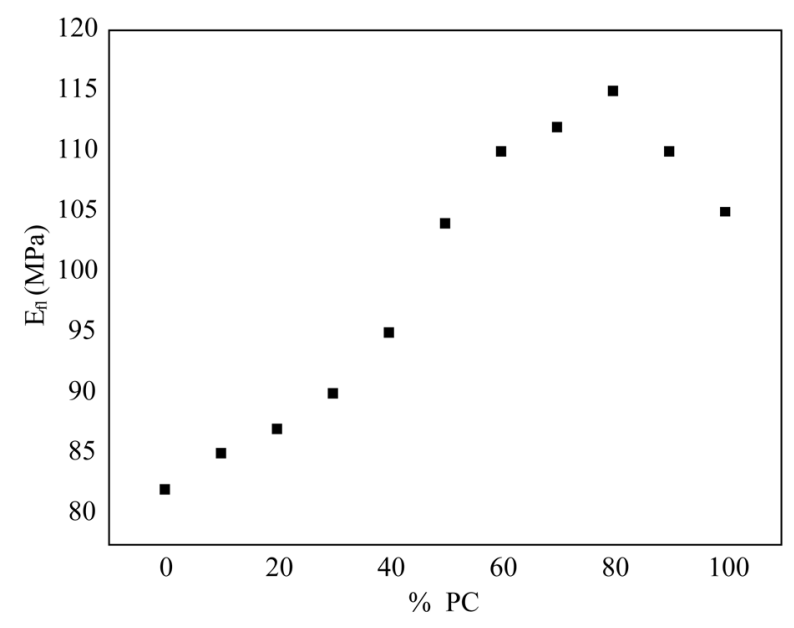

Figure 4. Flexural Modulus of PC/ABS blends as a function of the PC content.

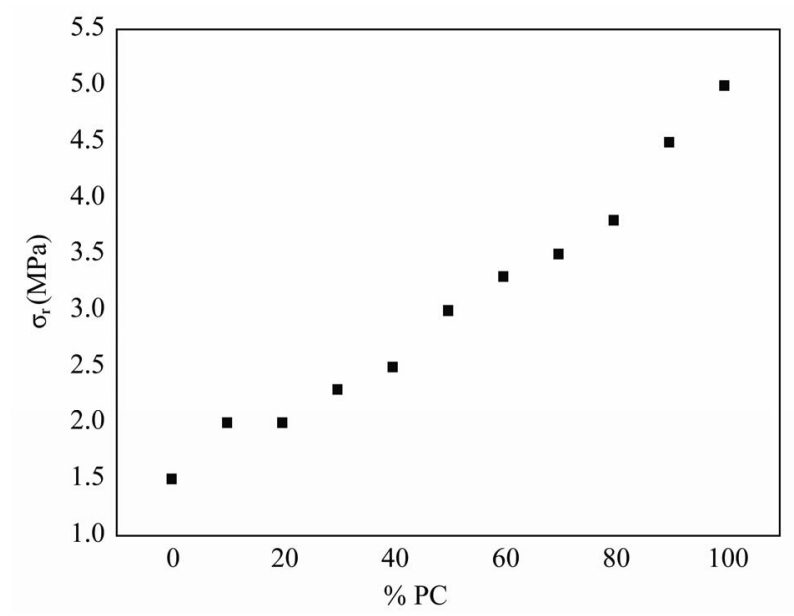

Figure 5. Flexural stress at break of PC/ABS blends as a function of the PC content.

ABS. In fact, an interesting observation has been made by Suarez et al. [7]: a sudden drop in the impact strength value occurred when a small amount of PC was added to the blends, the impact resistance character of PC did not offer any improvement in impact resistance to the ABS/ PC blends. Suarez et al. stated that the addition of PC in ABS generally increased the impact strength of the resulting $\mathrm{ABS} / \mathrm{PC}$ blends, but an inflection occurred in the ABS enriched ABS/PC blends.

Theoretically, in the ABS morphology, the butadiene rubber particles are dispersed in the Styrene Acrylonitrile (SAN) phase. The special properties of the ABS are thus created by rubber toughening, where fine particles of elastomer are distributed throughout the rigid matrix. The addition of PC minor amounts to the ABS results in the formation of triple phase morphology: the SAN continuous phase, the dispersed butadiene rubber particles in

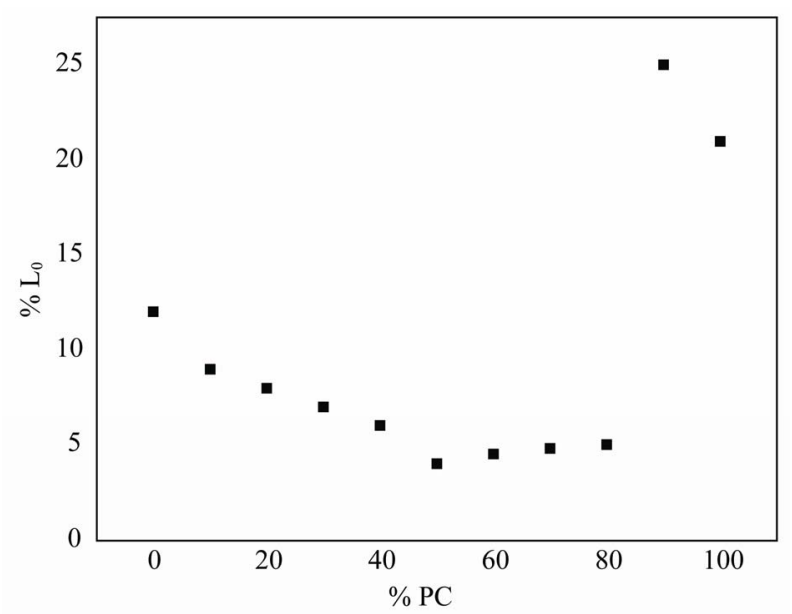

Figure 6. Flexural strain at break of PC/ABS blends as a function of the PC content.

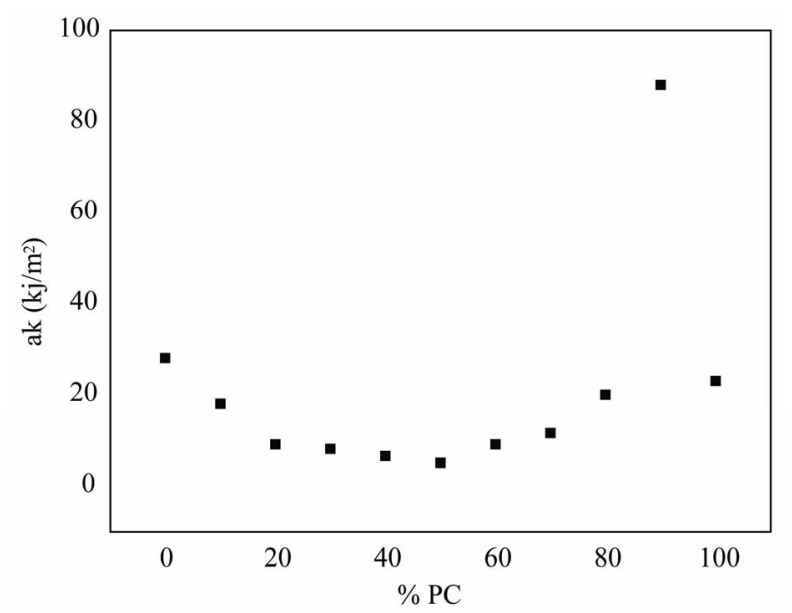

Figure 7. Impact strength of PC/ABS blends as a function of the $P C$ content.

SAN and the dispersed PC particles in SAN. However, as the PC content increases, it gradually becomes the continuous phase in the blend, whereas the ABS particles are the dispersed phase. The obtained results show that if the $\mathrm{PC}$ remains as disperse phase, it is not effective in initiating the yielding in the SAN continuous phase. On the contrary, when the ABS is in disperse phase, it is relatively more effective in initiating the yielding and in increasing the impact resistance of the blends [8].

\subsection{Hardness}

The hardness variation with the blend composition on PC/ABS blends (Figure 8) present two horizontal regions or "plateaux" for the matrices rich in $\mathrm{ABS}$ on one side, and for the matrices rich in $\mathrm{PC}$, on the other side. An increase in hardness with an increase of the PC content appears between the two "plateaux", due to the de- 


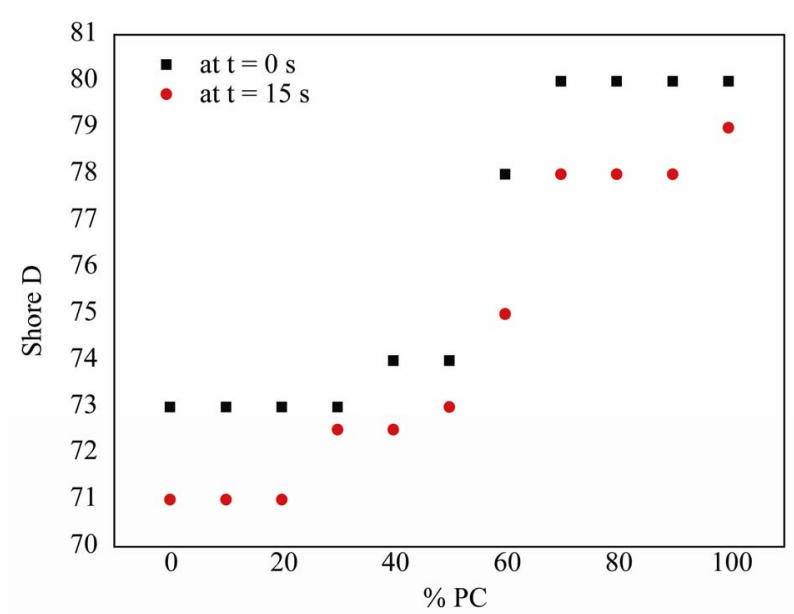

Figure 8. Hardness Shore D of PC/ABS blends as a function of the PC content.

crease in flexibility of the ABS chains. The hardness values found are lower than those cited in the literature because the phenomenon of surface degradation that takes place in the samples tested. The increase in hardness and the abrupt increase at higher proportion of PC can be explained by the phase inversion of $\mathrm{PC}$ from dispersed to continuous phase when its concentration in the blend was increased from $40 \%$ to $70 \%$.

\subsection{Heat Deflection and Vicat Temperature}

The HDT can be considered as a measure of the temperature at which certain creep compliance is reached after the polymer has been subjected to a standard temperature program. Measurements of the HDT of blends as a function of their composition can then be expected to give information on their phase topology. This possibility requires, of course, that the transition temperatures of the two components are sufficiently separated [9].

Figure 9 shows the HDT's measured for all the samples investigated. The curve of the binary PC/ABS system shows two different paces, indicating the occurrence of a phase inversion between $\mathrm{PC}$ and $\mathrm{ABS}$ at $50 \%$ of blend composition.

The PC/ABS polymer blends have found commercial utility. However, it is obvious that only a precise balance of polymer types and blend ratios can produce blends of high impact strength with a minimal sacrifice of the heat deflection temperature.

On the other hand, the values of the Vicat softening temperature drop steadily from that of pure $\mathrm{PC}$ to that of pure ABS (see Figure 9), forming a small plateau at 0 $10 \%$ PC. The complex shapes of these curves suggest some of the complexity of this system, which may involve partial solubility, coexistence of three or more phases, changing domain sizes, and other critical variables.

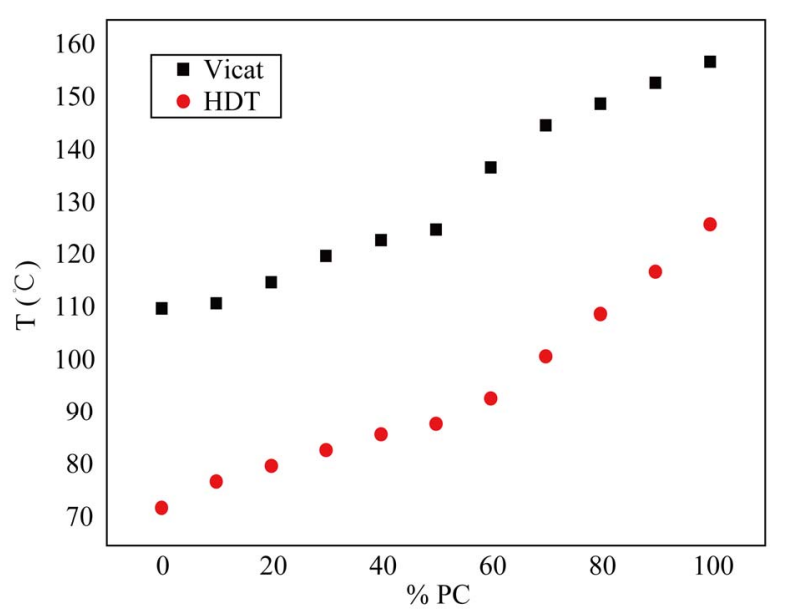

Figure 9. Heat deflection temperature HDT and Vicat softening temperature of PC/ABS blends as a function of the PC content.

Generally, gradual increases of the HDT and Vicat is observed as the PC content is increased.

\section{Morphology and FTIR Characterization}

The morphology of ABS/PC blends is complex and depends on the composition, the type of ABS and PC, the conditions of implementation and on the interfacial interaction. Thus, for ABS or PC content between 0 and $40 \%$ by weight, the minority phase is dispersed as nodules or fibrils in the matrix. For compositions near $50 \%$, the structure is co-continuous, that the ABS and PC phases are interconnected. Experimental results indicate that the impact strength of the ABS/PC blends is higher when the morphology is fibrillar or co-continuous [10-12].

In order to verify the above hypothesis, the morphology of the ABS/PC blends was examined with the help of a scanning electron microscope. Figure 10 shows micrographs of the fractured surfaces with respect to composition of the PC-rich blend and ABS-rich blend.

For the blends containing $90 \% \mathrm{PC}$, we can see that the ABS phase appears as spherical inclusions in the PC phase matrix and the blend look more uniform. For the blend containing $40 \% \mathrm{PC}$, the PC phase is dispersed as nodules in the ABS matrix and the dispersion is irregular.

The IR spectrums (see Figure 11) reveal the absence of oxidized species around $3200-3300 \mathrm{~cm}^{-1}$ in the homopolymers, this means that the polymers do not undergo significant degradation during the implementation. This reveals also the very good heat resistance of PC. For the blend of (90/10) PC/ABS, the spectrum show no significant difference compared to the spectra of pure PC and pure $\mathrm{ABS}$, that means that there is no chemical reaction between $\mathrm{PC}$ and ABS. Thus the optimum properties 


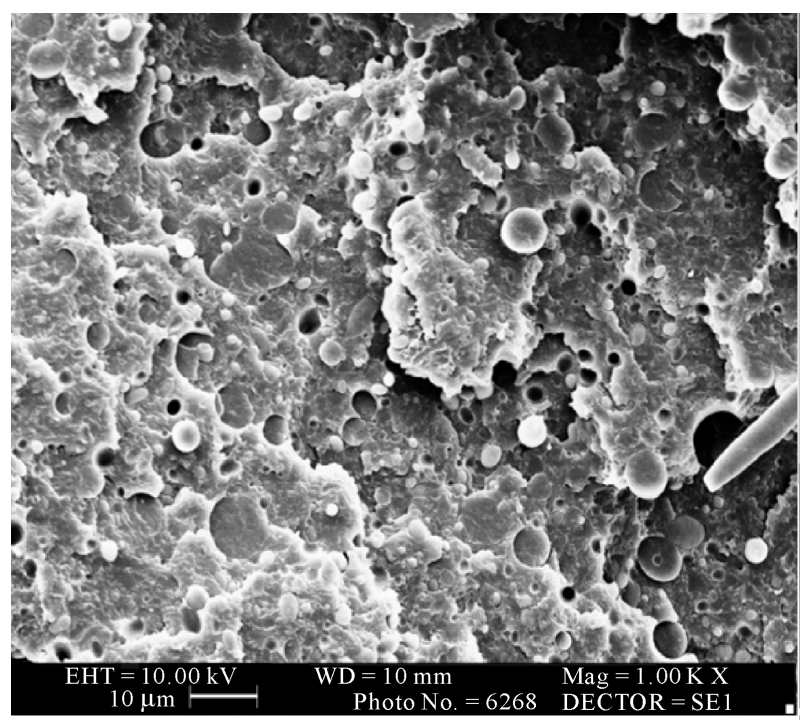

(a)

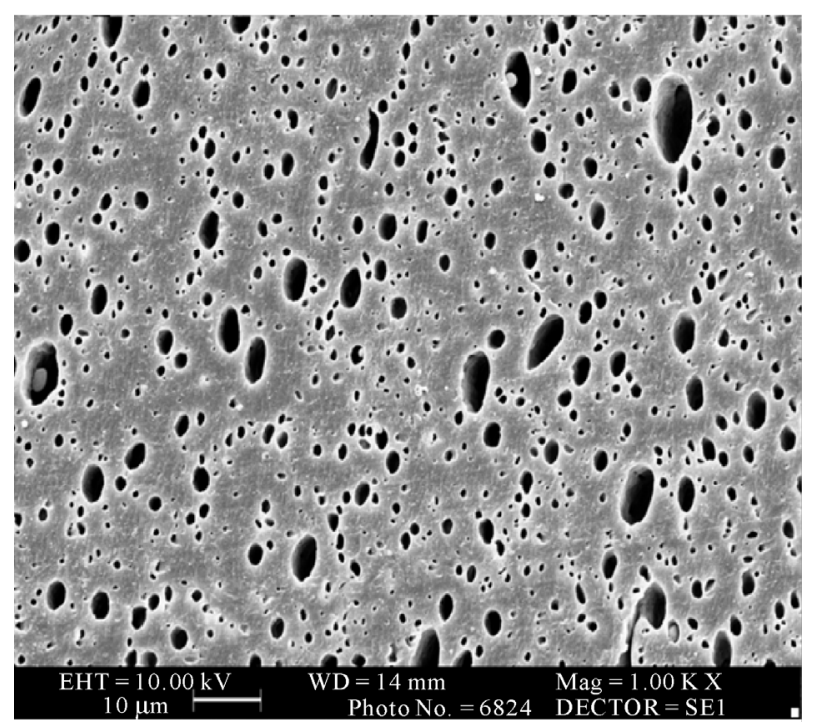

(b)

Figure 10. Scanning electron micrographs of fractured surfaces of: (a) (90/10) PC/ABS blend and (b) etched surface of (40/60) PC/ABS blend.

obtained with a rate of $90 \%$ PC can be attributed to partial miscibility between PC and ABS.

\section{Conclusions}

In this work we have systematically studied a number of properties of PC/ABS blends as a function of composition. Ultimate properties, such as impact strength and toughness, present attractive enhancement for a $90 \%$ PC composition. Indeed, despite contradictory trends cited in several research studies, all authors always get an impact strength of blends with high rates of PC. The addition of a few PC to the ABS leads in general, to a resilience of

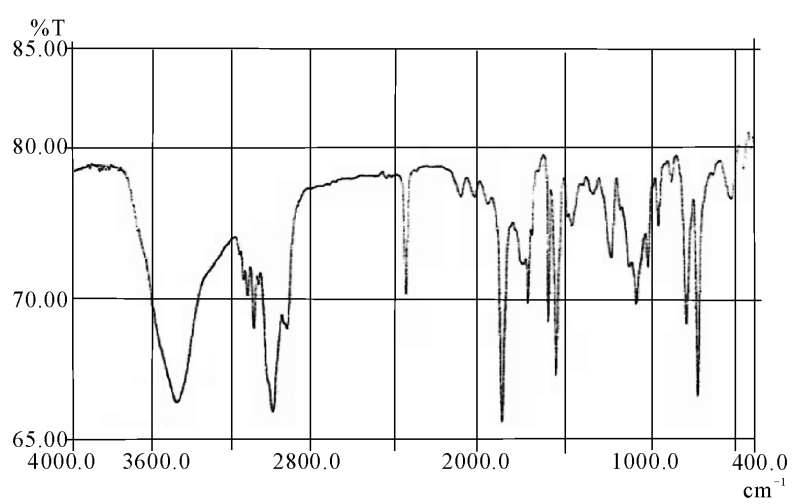

(a)

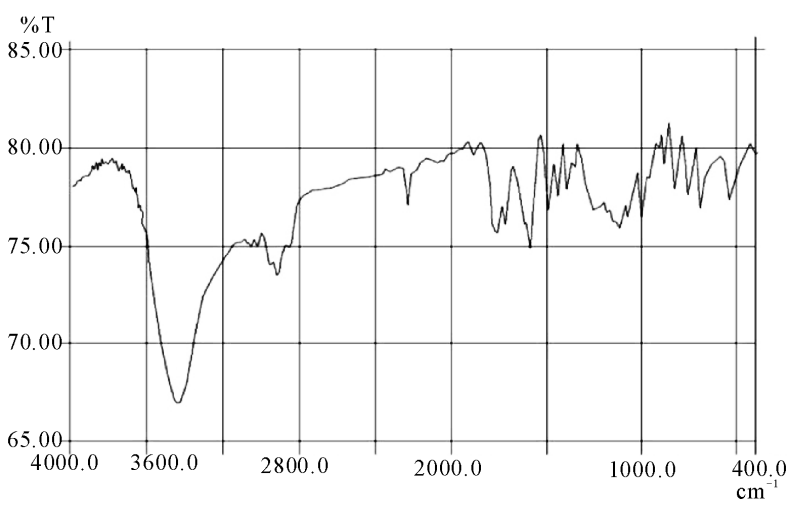

(b)

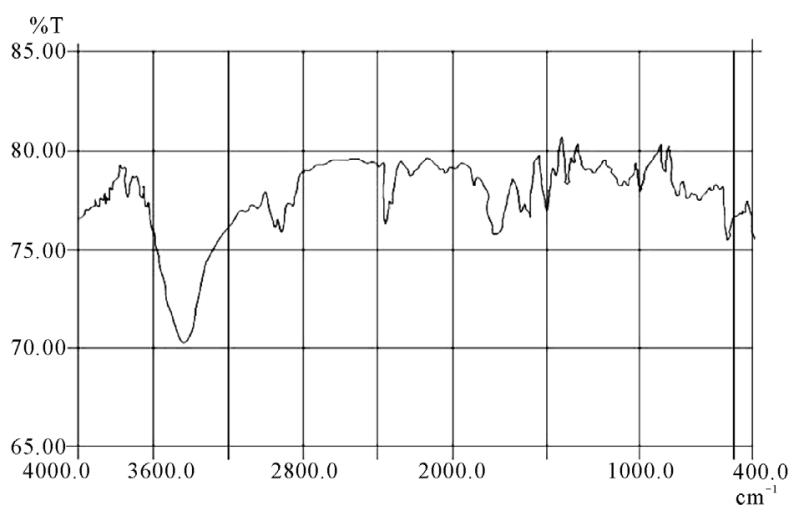

(c)

Figure 11. FTIR Spectrum of (a) pure ABS, (b) (90/10) PC/ ABS Blend and (c) pure PC.

blends less than that of ABS. The rate of PC for which the resilience of the mixture is maximum varies according to the authors. The elastic modulus, the heat deflection temperature and the Vicat softening temperature are intermediate between those of the components for any blend composition. These two last properties show the occurrence of an inversion phase for the 50/50 composition. The morphology of the PC/ABS blend was found to be co-continuous. The results obtained in this study show 
some interesting aspects that may be of practical application in case of blends with high PC content, the mixtures typically have a partial miscibility (or intermixing miscibility window) at $90 \% \mathrm{PC}$ and the properties are optimal. In case of blends with low PC content, the decrease in properties is an important disadvantage for the practical application of these blends.

\section{REFERENCES}

[1] L. A. Utracki, "Polymer Blends Handbook," Kluwer Academic Publisher, Amsterdam, 2002.

[2] F. Nordgren and M. Nyquist, "FE-Modelling of PC/ ABS-Experimental Tests and Simulations," Master's Dissertation, Division of Solid Mechanics, Lund University, Lund, 2006.

[3] L. A. Utracki and B. D. Favis, "Polymer Alloys and Blends," In: P. N. Cheremisinoff, Ed., Handbook of Polymer Science and Technology, Marcel Dekker, Inc., New York, 1989, pp. 121-201.

[4] A. Pavan, T. Riccò and M. Rink, "High Performance Polymer Blend," Materials Science and Engineering, Vol. 48, No. 9, 1981, pp. 9-15. doi:10.1016/0025-5416(81)90060-4

[5] R. D. Deanin and R. R. Geoffroy, "Major Thermoplastic Polyblends: Compatibility and Practical Properties," Organic Coating and Plastics Chemistry, Vol. 37, No. 1, 1977, pp. 257-262.

[6] J. M. Martínez, J. I. Eguiazábal and J. Nazábal, "Phase Behavior of Mechanical Properties of Injection Molded
PET/Par Blends," Journal of Applied Polymer Science, Vol. 45, No. 7, July 1992, pp. 1135-1143. doi:10.1002/app.1992.070450702

[7] H. Suarez, J. W. Barlow and D. R. Paul, "Mechanical properties of ABS/polycarbonate blends," Journal of Applied Polymer Science, Vol. 29, No. 11, November 1984, pp. 3253-3259. doi:10.1002/app.1984.070291104

[8] A. Hassan and W. Y. Jwu, "Mechanical Properties of High Impact ABS/PC Blends - Effect of Blend Ratio," Polymer Symposium, Kebangsaan Ke-V Hotel Residence, August 2005, pp. 23-24.

[9] J. Bastida, I. Eguiazábal and J. Nazábal, "The Vicat Softening Temperature as a Method to Assess the Phase Behaviour of Amorphous Polymer Blends," Polymer Testing, Vol. 12, No. 3, 1993, pp. 233-242. doi:10.1016/0142-9418(93)90005-A

[10] M. L. Barthes, "Régénération d'ABS et de PC Issus de DEEE sous Forme d'Alliages de Polymères Techniques ou de Nanocomposites," Ph.D. Dissertation, Mechanic and Engineering, Doctoral School of Physical Sciences for Engineering, Bourdeaux, 2010.

[11] M. M. K. Khan, R. F. Liang, R. K. Gupta and S. Agarwal, "Rheological and Mechanical Properties of ABS/PC Blends," Korea-Australia Rheology Journal, Vol. 17, No. 1, 2005, pp. 1-7.

[12] T. Matchimapiro, P. Sornthummalee, T. Pothisiri and S. Rimdisit, "Impact Behaviors and Thermomechanical Properties of TPP-Filled Polycarbonate/Acrylonitrile-Butadiene-Styrene Blends," Journal of Metals, Materials and Minerals, Vol. 18, No. 2, 2008, pp. 187-190. 